\title{
Application of Artificial Neural Network for Clutter Rejection
}

\author{
Priyabrata Karmakar ${ }^{1}$, Sourav Dhar ${ }^{2}$, Mithun Chakraborty ${ }^{3}$, Tirthankar Paul ${ }^{4}$ \\ ${ }^{1,3}$ Electronics \& Communication Engineering Department, Surendra \\ Institute of Engineering \& Management, Siliguri, India \\ ${ }^{2}$ Electronics \& Communication Engineering Department, Sikkim Manipal \\ Institute of Technology, Sikkim, India \\ ${ }^{4}$ Electronics \& Communication Engineering Department, Swami Vivekanada \\ Institute of Science \& Technology, India \\ p.karmakar87@gmail.com
}

\begin{abstract}
This paper deals with application of Artificial Neural Network (ANN) for Radar Clutter rejection, function approximation method of supervised ANN is applied here using back propagation algorithm. The database used for training and testing the ANN has been collected from simulating a moving vehicle in MATALAB(version 7.9) to obtain the RCS values at range and cross-range profiles. This work is validated by comparing received signal after clutter rejection with the received signal in no clutter condition.
\end{abstract}

Index Terms - Artificial Neural Networks, Radar, Clutter

\section{I.INTRODUCTION}

Radar returns are produced from nearly all surfaces when illuminated by radar. Therefore, in competition with the return from a target, there are many sources of unwanted signals. Unwanted signals in search radar are generally described as noise and clutter. Clutter is the term used and includes ground returns, sea returns, weather, buildings, birds and insects. Wei You et al [4] proposed a fast clutter rejection method for ultrasound color flow imaging based on the first-order perturbation as an efficient implementation of eigendecomposition and the method was verified by simulated data. R. Vicen-Bueno et al [5] proposed an estimation of the ship size using an ANN-based clutter reduction system followed by a fixed threshold, high clutter reduction rates were achieved using 1dimensional (horizontal or vertical) integration modes, although inaccurate ship width estimations were achieved. These estimations were improved using a 2dimensional (rhombus) integration mode. Proposed system was compared with a CA-CFAR system, denoting a great performance improvement and a great robustness against changes in sea clutter conditions and ship parameters, independently of the direction of movement of the ocean waves and ships. P.K Verma et al [6] applied through wall imaging (TWI) technique for clutter rejection, firstly observed data are preprocessed for imaging and then different types of clutter reduction techniques like Principal Component
Analysis (PCA), Independent Component Analysis (ICA), Factor Analysis (FA) and Singular Value Decomposition (SVD) have been applied, and results were analyzed. Signal to noise ratio (SNR) of the final images had been computed to compare the results and know the effectiveness of individual clutter removal techniques. S.L Durden et al [7] found that Doppler filtering can significantly reduce the surface return, bringing surface clutter to acceptable levels. Keerthi Ram et al [8] presented a new approach for automatic microaneurysm detection from digital colour fundus images, they formulate MA detection as a problem of target detection from clutter, where the probability of occurrence of target is considerably smaller compared to the clutter. Wei You et al [9] proposed a clutter rejection method based on the recursive eigendecomposition algorithm. In this method, the current eigenvector matrix of the ultrasound echo correlation matrix, which will be used to construct the clutter subspace, is determined by previous eigenvector matrices and the current input. Jin Yonggao et al [10] proposed new clutter rejection method named "nonlinear projection" to improve the SNR of the target as sea clutter masks some weak target signals, clutter modeling is done as a nonlinear deterministic dynamical system. After approximating the multidimensional reconstruction of the clutter by a low-dimensional attractor, projections onto this attractor can separate the clutter from other components. Real sea clutter, simulated target data and real target data are used to show that a nonlinear clutter rejection method is a promising technique to suppress sea clutter and enhances target detection. The definition of clutter depends on the function of the radar. Since vehicles usually move much faster than weather or surface slow moving targets, velocitysensitive radar can eliminate unwanted clutter from the radar indicator. In Radar systems Artificial Neural Network can be applied to reject unwanted Clutter by applying the concepts of Supervised artificial Neural network using Back-Propagation as the learning algorithm. Rest of the paper is organized as section II describes how database is made for the experiment, in section III,IV and V details of Artificial Neural 
Network, ANN architecture and pre and post processing of data are given respectively, Clutter model and Clutter rejection described in section VI and section VII respectively, in section VIII results are given and finally section IX concludes the paper.

\section{DATABASE}

To collect data for training the network we have simulated a light vehicle using their structure in MATLAB to get the RCS values using the below figure,

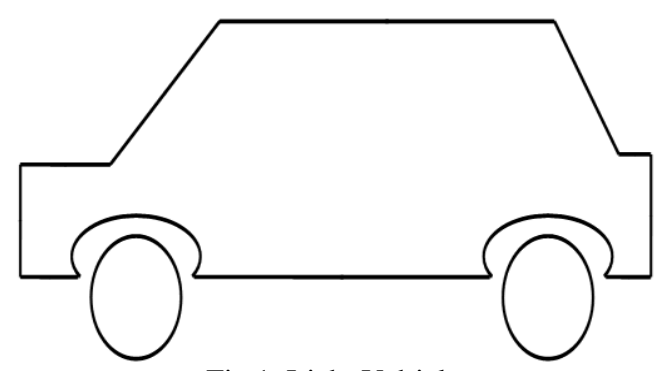

Fig 1. Light Vehicle

Above figure is the side view of the corresponding vehicle. we can consider this side view image of the target as the combination of several rectangular, triangular and circular flat plate and if the RCS values for those flat plates can be calculated then it is easily possible to calculate the RCS values of our target of interest, RCS values for those flat plates can be given as in [1]. So the clutter-free RCS values for light vehicle at range and cross-range profile are obtained, for collection of range profile data we have taken twenty different frequency values within the range of ( $22 \mathrm{GHz}$ to $29 \mathrm{GHz}$ ), and to collect cross-range profile data twenty different aspect angle values are taken within the range of ( 0 to 8 radian). This values of frequency and aspect angles are taken as input parameters to train the network whereas corresponding RCS values are taken as target to the network to be trained.

For simulating the network we have taken different values frequency and aspect angles which values are unseen to the network and those values fed in to the network as input during simulation of the network, after simulating the network we got the estimated RCS values for those input parameters.

\section{ARTIFICIAL NEURAL NETWORK}

There is no universally accepted definition of neural network, in [2] neural network is a massively parallel distributed processor that has a natural propensity for storing experimental knowledge and making it available for use, it resembles brain in two ways i) knowledge is acquired by the network through learning process and ii) Interneuron connection strengths known as synaptic weights are used to store the knowledge. Learning in NN means a dynamic process which modifies the weights of the network in some desirable way. There are two types of learning algorithms in ANN, supervised and unsupervised learning. In supervised learning the network is presented with both inputs and targets whereas in later it is presented with only inputs. The learning mechanism adopted by us is supervised and based on back propagation algorithm. Here the MSE between network output and the target is back propagated to the previous layers of the $\mathrm{NN}$ to minimize error by adjusting weights, the error minimization and weight updating has been implemented using gradient descent algorithm.

\section{ANN ARCHITECTURE}

We have chosen a multi layer feed forward neural network architecture [ [ $\left.\begin{array}{llll}2 & 25 & 25 & 1\end{array}\right]$ consisting of two hidden layers each of which are having twenty five neurons, input and output layers are having two and one neuron respectively, linear activation function is applied to the input layer neurons whereas non-linear tan-sigmoid activation function is applied to hidden layer neurons. 'newfit' function from the neural network toolbox of MATLAB is used here for the training of ANN.

Table I. ANN architecture and parameters

\begin{tabular}{|c|c|}
\hline Number of neurons in input layer & 2 \\
\hline Number of hidden layers & 2 \\
\hline $\begin{array}{c}\text { Number of neurons in each hidden } \\
\text { layers }\end{array}$ & 25 \\
\hline Number of neurons in output layers & 1 \\
\hline Input layer activation function & Linear \\
\hline Hidden layer activation function & $\begin{array}{l}\text { Tan- } \\
\text { sigmoid }\end{array}$ \\
\hline Output layer activation function & $\begin{array}{c}\text { Tan- } \\
\text { sigmoid }\end{array}$ \\
\hline
\end{tabular}

\section{PRE PROCESSING AND POST PROCESSING OF DATA}

Pre-processing of input or training data and target data includes normalization of the data within a specific range as the values in the input matrix and target vector are large enough, it may increase the complexity of the network. So it is preferable to normalize the values in the input matrix and target vector to map the dataset into a small range, There are many statistical data normalization methods available which can be applied to ANN, here the equation used for the normalization is given below,

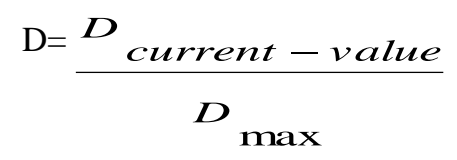

Where, 
D current-value $\rightarrow$ it is the element of input matrix/target vector at a particular instant.

$\mathrm{D}_{\max } \rightarrow$ maximum value of elements in the input matrix/target vector.

Post-processing of simulated data includes denormalization of the data to get back the data in it's original range. Equation used for the de-normalization is given below,

$$
\mathrm{S}=S \text { current }- \text { value }{ }^{\times D} \max
$$

Where,

$$
S_{\text {current-value }} \rightarrow \text { it is the element of }
$$
simulated data vector at a particular instant.

\section{CLUTTER MODEL}

The model for clutter used here is,

$$
\sigma_{\text {clutter }}=\frac{\sin (\theta) \cos (\theta)}{\sec (\theta) \tan (\theta)} \times \frac{\sqrt{f}}{2 \pi}
$$

Where,

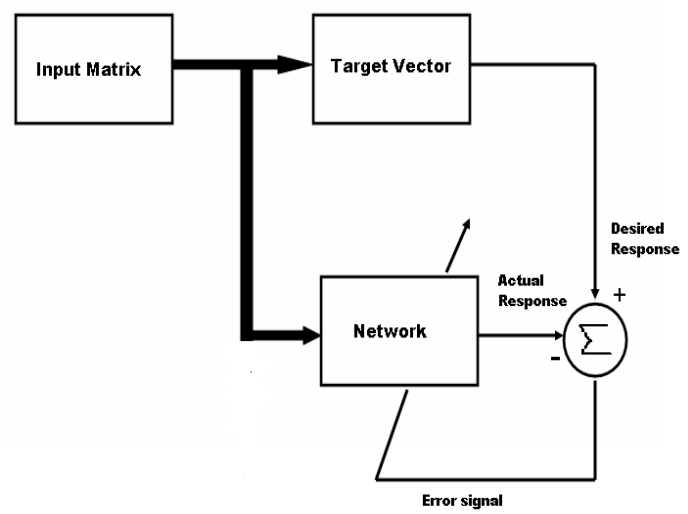

Fig 2. Arrangement for Network training

\section{RESULTS}

At first neural network is trained using the training data, initial training parameters are listed in the table below

TABLE II. Initial training parameters

\begin{tabular}{|c|c|}
\hline Learning rate & 0.1 \\
\hline Time & infinite \\
\hline Epoch & infinite \\
\hline Error goal & $10^{-6}$ \\
\hline
\end{tabular}

$\theta \rightarrow$ Aspect angle

$f \rightarrow$ Frequency

Clutter RCS are taken in range and cross-range profile for the same values of frequency and aspect angle as for simulating the network, we named this as 'Clutter Signal'.

\section{CLUTTER REJECTION}

To reject Clutter first RCS values for Light Vehicle are stored in range and cross-range profile for the frequency and aspect angle as for simulating the network, we named this as 'Original Signal', in Radar receiver 'Original Signal' is received with added 'Clutter Signal' we named this as 'Cluttered Received Signal'

Cluttered Received Signal $=$ Original Signal + Clutter Signal

'Estimated Signal' is obtained by simulating the trained network as mentioned in article II. By comparing 'Estimated Signal' with 'Cluttered Received Signal'added 'Clutter Signal' can be rejected from 'Cluttered Received Signal'. Added Clutter = Cluttered Received Signal - Estimated Signal

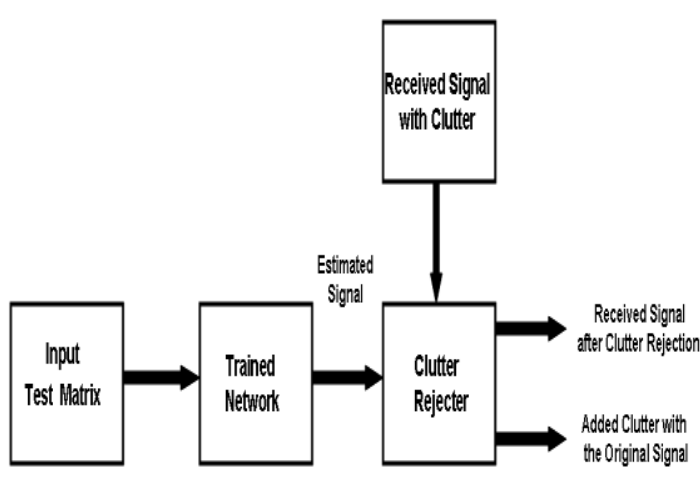

Fig 3. Arrangement for Clutter Rejection

Proper network training is justified by analysis of Performance Plot and Regression Plot

After training network parameters are listed in the table below

TABLE III. Network parameters after training

\begin{tabular}{|c|c|}
\hline Total time for training & $1064 \mathrm{sec}$ \\
\hline Total epochs run & 168 \\
\hline $\begin{array}{c}\text { Overall Performance } \\
\text { (Error gradient) }\end{array}$ & $8.59 \times 10^{-5}$ \\
\hline
\end{tabular}




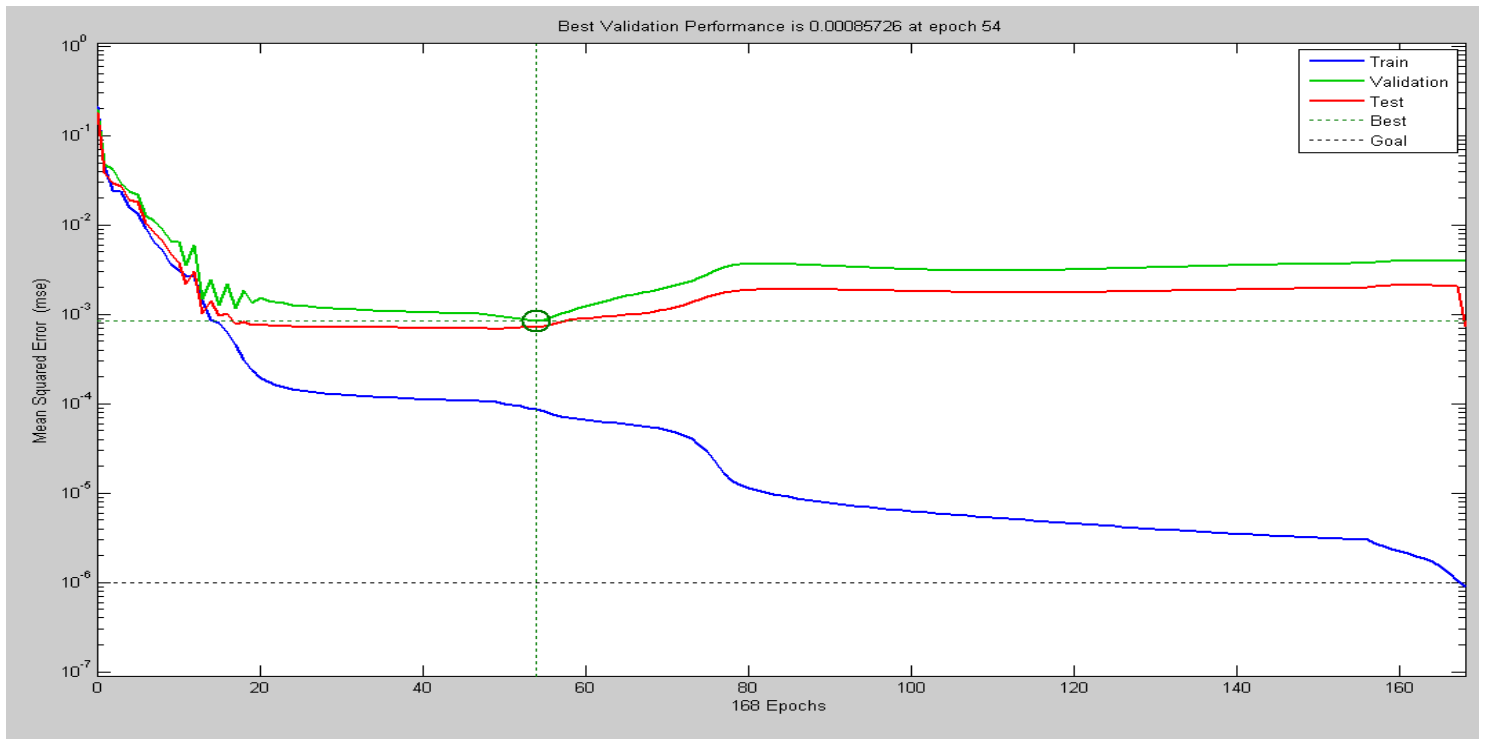

Figure 4. Performance Plot

Table IV. Error gradient reached during different phases of network training

\begin{tabular}{|l|l|}
\hline During training & $10^{-6}$ \\
\hline During validation & $8 \times 10^{-2}$ \\
\hline During testing & $2 \times 10^{-3}$ \\
\hline Overall & $8.59 \times 10^{-5}$ \\
\hline
\end{tabular}

From the performance plot and table IV it is clear that error goal reached during training and overall performance is also satisfactory.
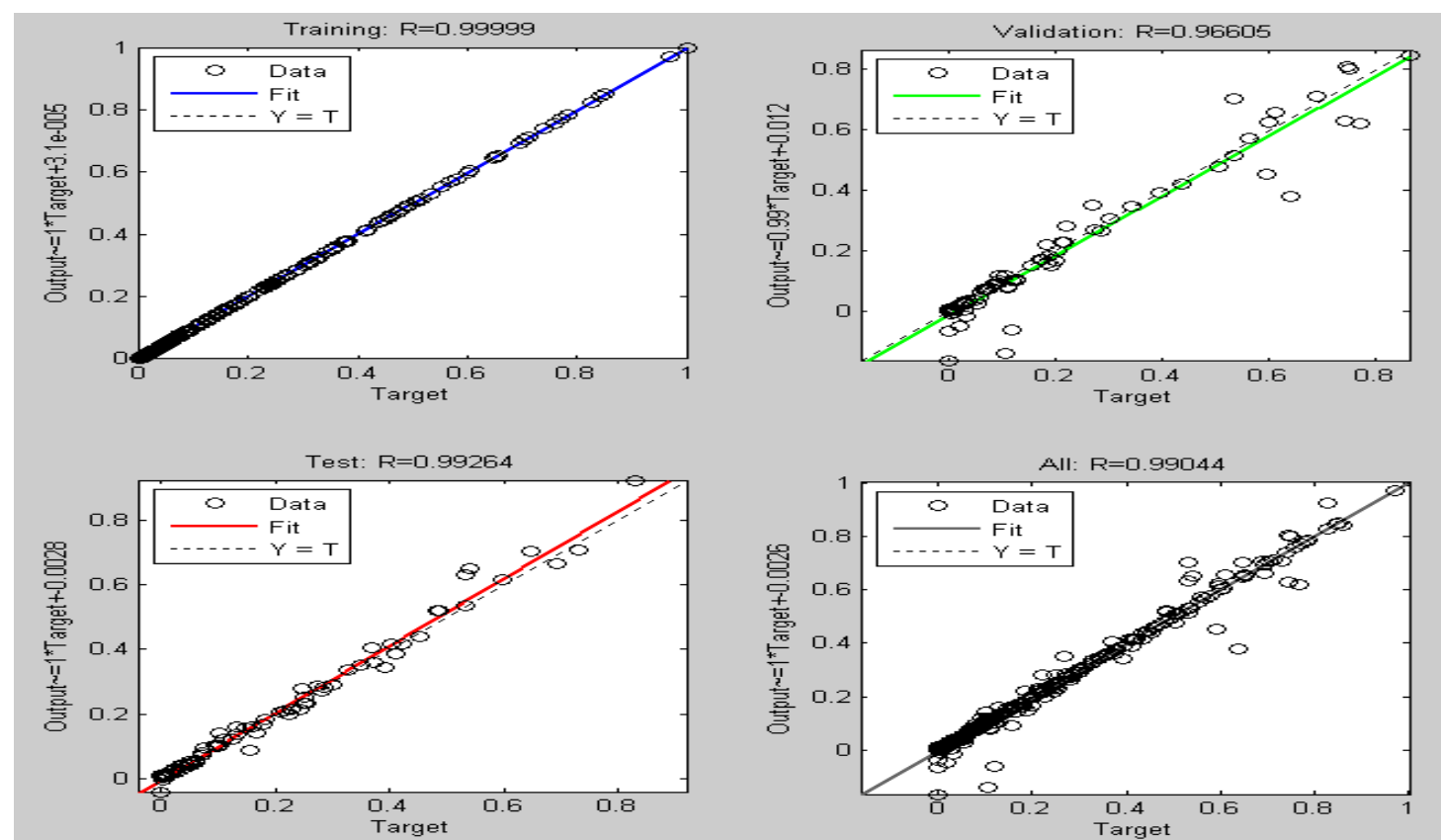

Figure 5. Regression Plot

Table V. Correlation coefficient ' $\mathrm{R}$ ' for Regression analysisreached during different phases of network training

\begin{tabular}{|c|c|}
\hline During training & 0.9999 \\
\hline During validation & 0.96605 \\
\hline During testing & 0.99264 \\
\hline Overall & 0.99044 \\
\hline
\end{tabular}

Regression Plot is to perform a linear regression between the network outputs and the corresponding targets, from the Regression plot and table $\mathrm{V}$ it is clearly seen that the output tracks the targets very well for training, testing, and validation, and the $\mathrm{R}$-value is over 0.99044 for the total response. 
After the network training is finished, using that trained network clutter is being rejected

Original Signal without Clutter environment, Clutter Signal, Received Signal with added Clutter, Received
Signal after Clutter rejection, Signal to Clutter ratio are shown in figures 6 to 11 .

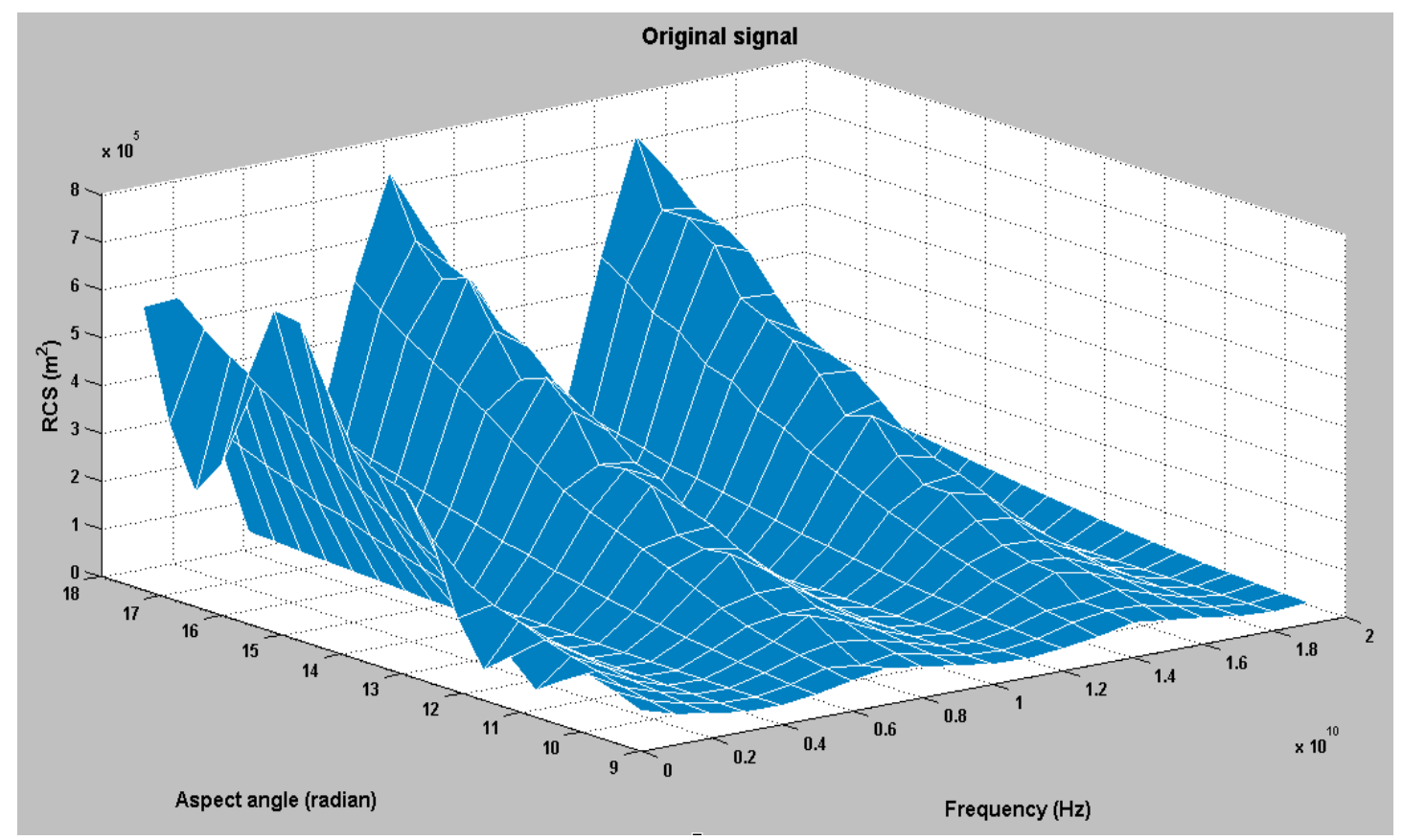

Figure 6. Original target returned signal in Clutter free environment

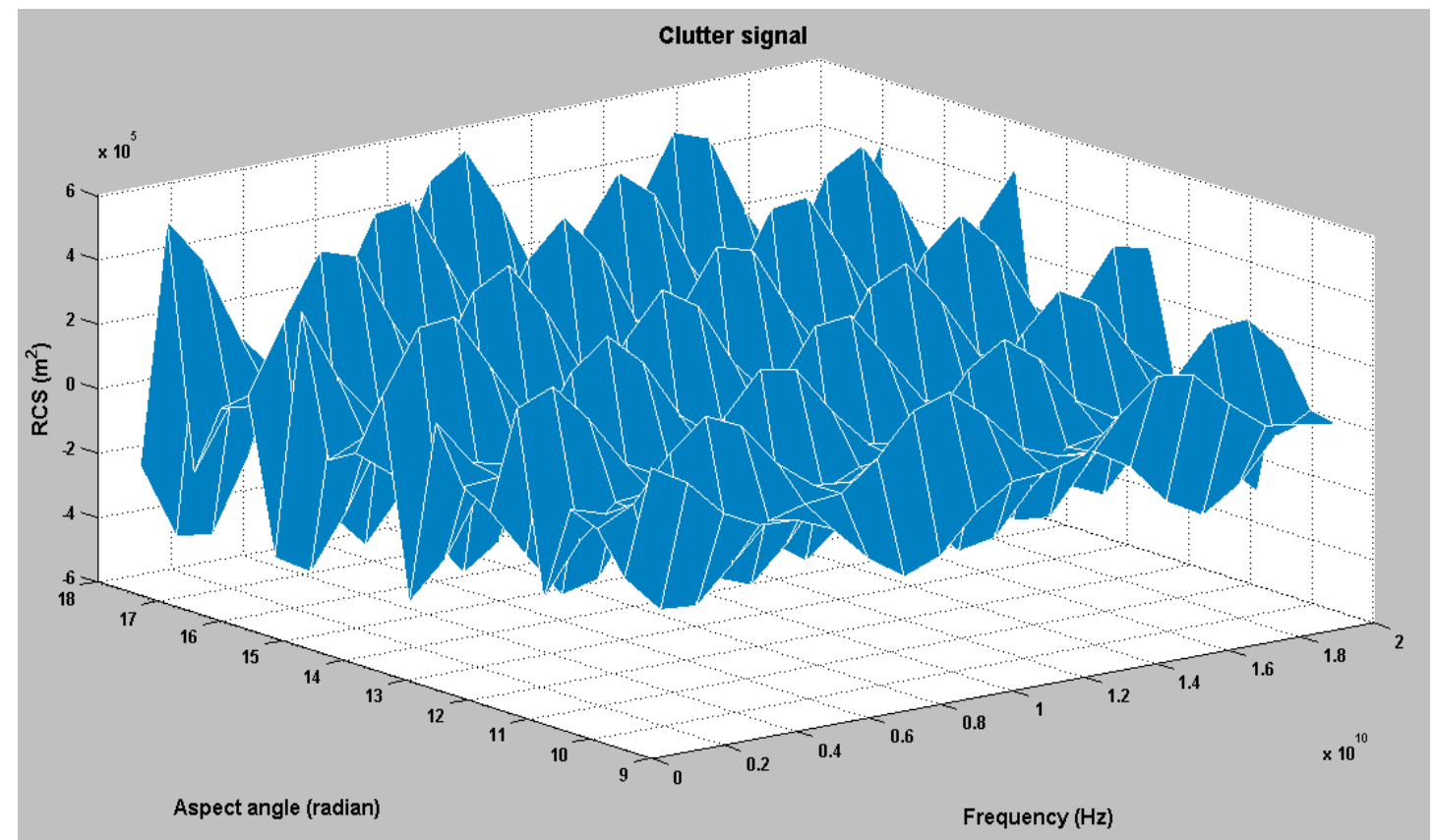

Figure 7. Clutter to be added with Original Signal 


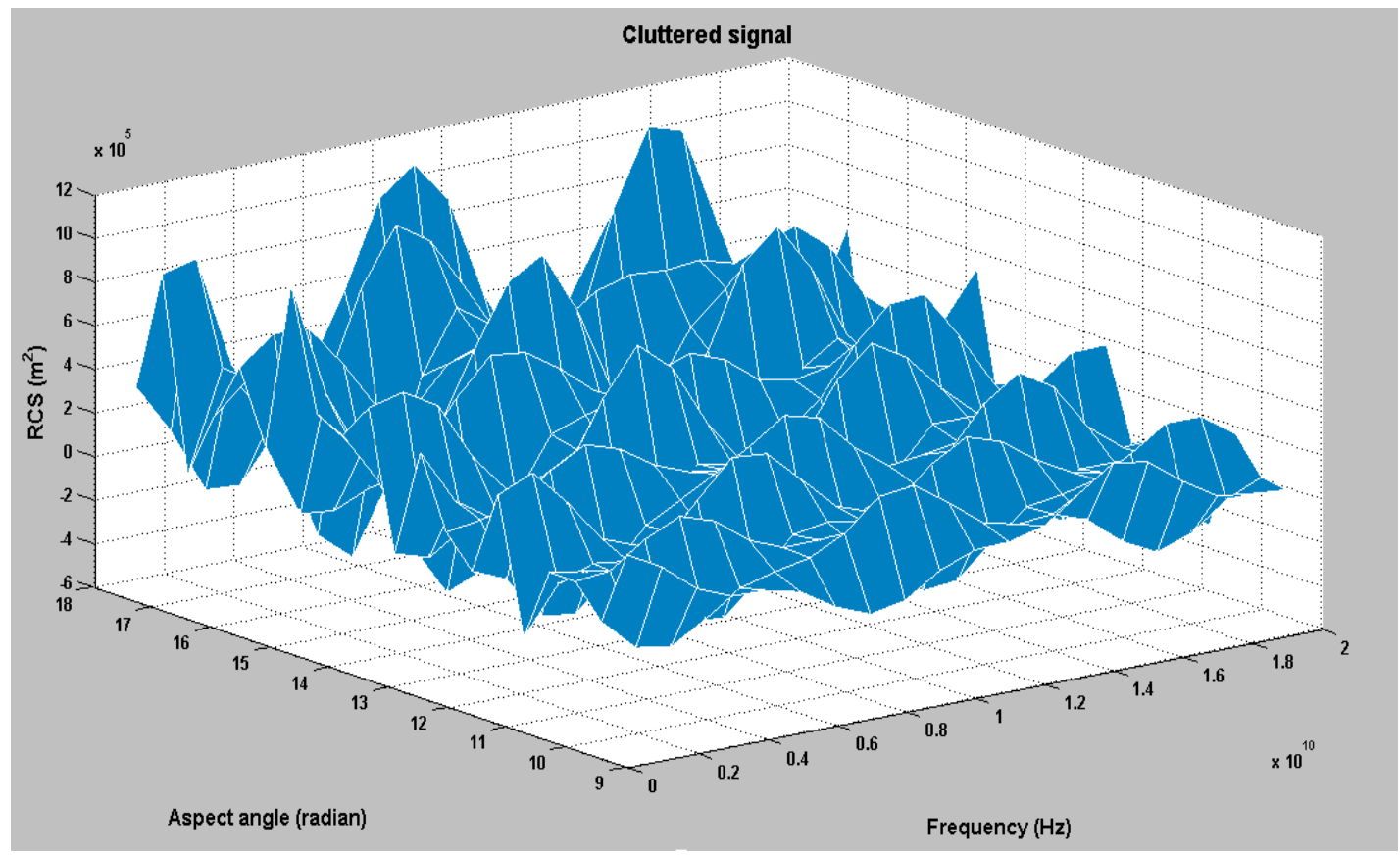

Figure 8. Cluttered Received Signal

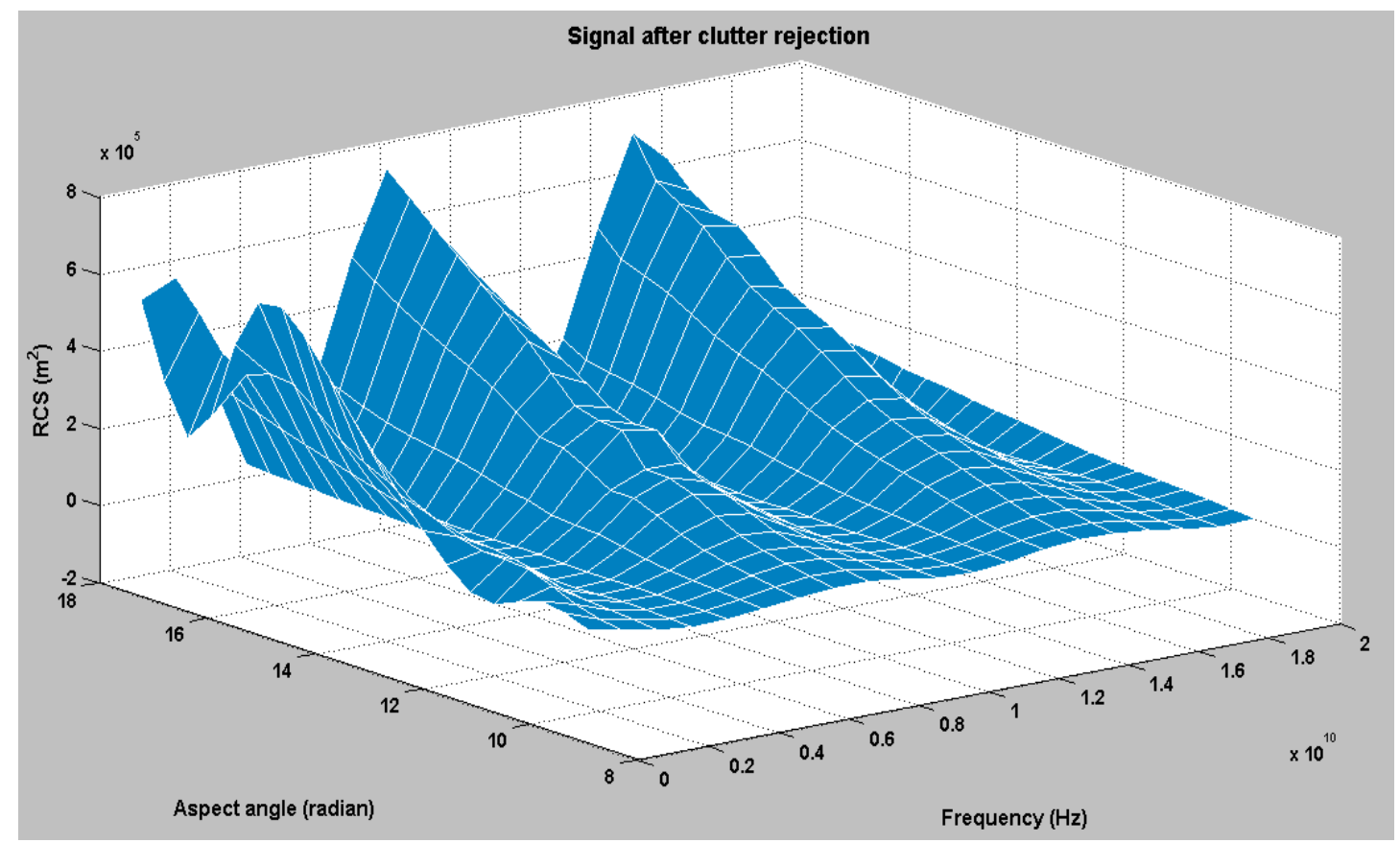

Figure 9. Clutter rejected Received Signal 


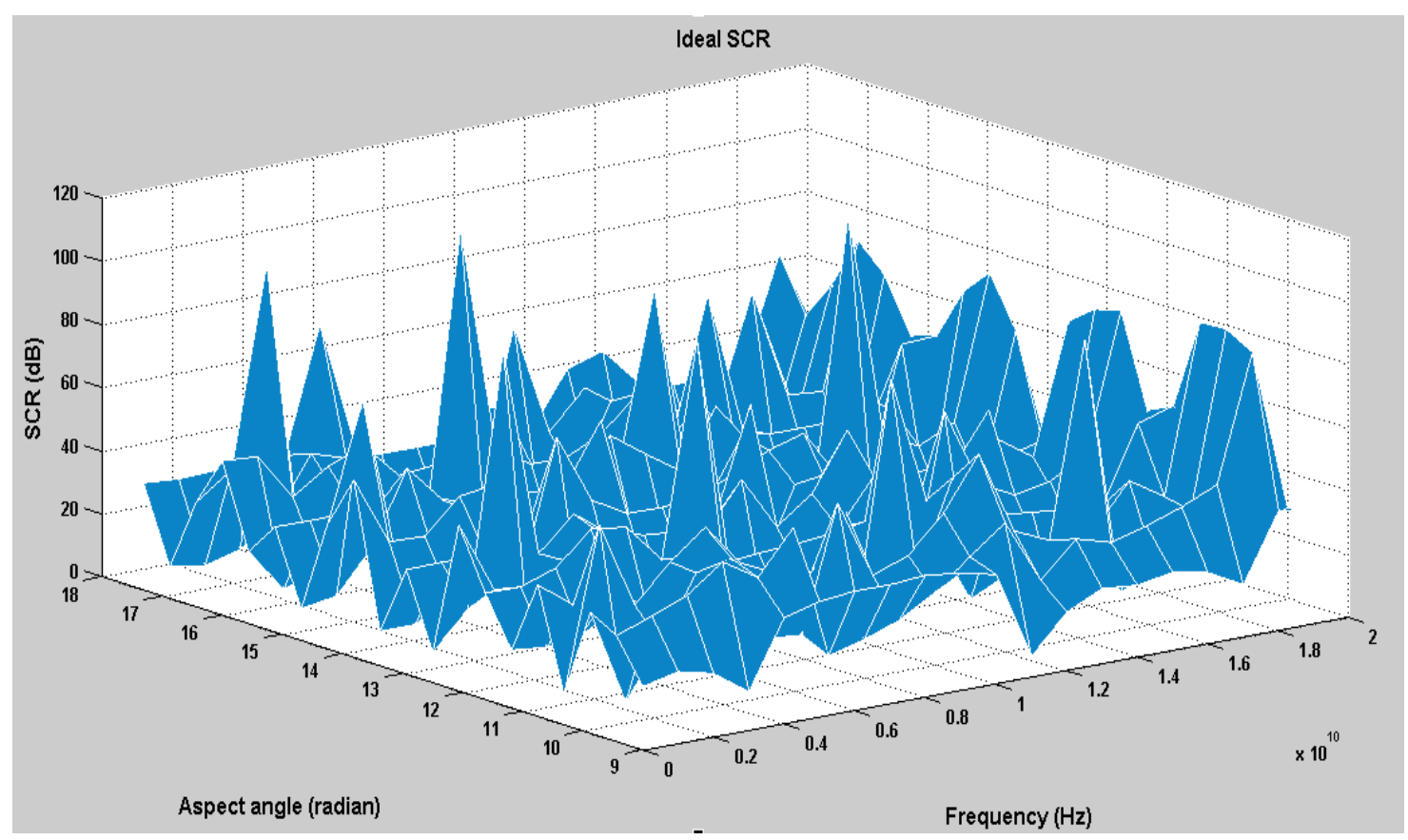

Figure 10. Ideal Signal Clutter ratio

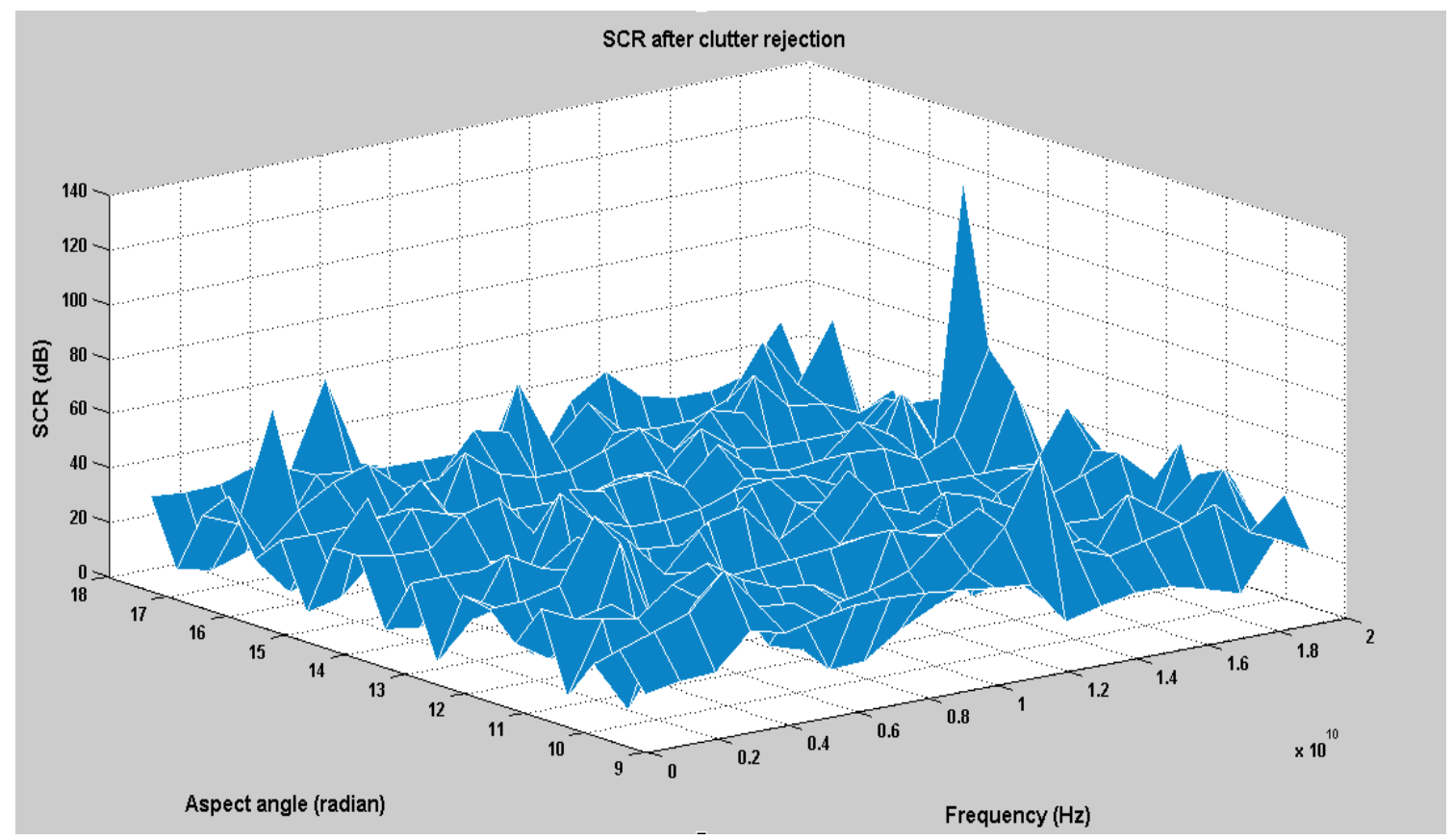

Figure 11. Practically observed Signal to Clutter ratio

\section{IX.CONCLUSION}

It can be observed that signal in figure 6 would be received if there is no clutter addition from the environment, but it is not possible in real scenario, signal in figure 9 is the received signal in practical case, by comparing both the figure it can be said that reception of signal is almost proper after clutter rejection, only some little variations are there when comparing Clutter rejected received signal with its counterpart in Ideal case. From figure 10 and figure 11 it can be noticed that Signal to Clutter ratio (SCR) in practical case has little variations comparing to Ideal case SCR. So we can conclude that ANN is trained properly which is efficiently used for clutter rejection and this concept can be implemented in Radar systems for proper reception of signals from aircrafts, ground moving targets and sea based targets.

\section{REFERENCES}

[1] Bassem R. Mahafza (2000), "Radar Systems Analysis and Design Using MATLAB", article 12.1, CHAPMAN \& HALL/CRC 
[2] Simon Haykin, "Neural Networks- a comprehensive foundation",Prentice Hall India, 1999

[3] E.Hanusa, D.Krout, M.Gupta,"Clutter Rejection by Clustering Likelihood-Based Similarities", 2011 Proceedings of the 14th International Conference on Information Fusion (FUSION), ISBN: 978-14577-0267-9,5-8 July,2011.

[4] W.You,Y.Wang, "A fast algorithm for adaptive clutter rejection in ultrasound color flow imaging based on the first-order perturbation: a simulation study", IEEE Transactions on Ultrasonics, Ferroelectrics and Frequency Control (2010),Vol.57,pp.1884-1889,2010.

[5] R. Vicen-Bueno et al, " Artificial Neural NetworkBased Clutter Reduction Systems for Ship Size Estimation in Maritime Radars", EURASIP Journal on Advances in Signal Processing, Vol.2010, doi:10.1155/2010/380473.

[6] P. K. Verma, A. N. Gaikwad, D. Singh, and M. J. Nigam, "Analysis of clutter reduction techniques for through wall imaging UWB range", Progress In Electromagnetics Research B, Vol.17, pp. 29-48, 2009.

[7] S. L. Durden , S. Tanelli, "Application of Clutter supression methods to a geostationary weather radar concept", Progress In Electromagnetics Research Letters, Vol. 8,pp. 115-124, 2009.

[8] Keerthi Ram, Gopal Datt Joshi, Jayanthi Sivaswamy, "A Successive Clutter-Rejection based Approach for Early Detection of Diabetic Retinopathy", IEEE transactions on Biomedical Engineering, TBME-00468-2010-R1.

[9] Wei You, Yuanyuan Wang,"Adaptive Clutter Rejection for Ultrasound Color Flow Imaging Based on Recursive Eigen decomposition", IEEE Transactions on Ultrasonics, Ferroelectrics and Frequency Control (2009), vol. 56, no. 10, October 2009

[10] Jin Yonggao,Dong Huachun,Quan Taifan, "HF radar sea clutter rejection by nonlinear projections",IEEE Journal of Systems Engineering and Electronics,Volume: 16, Issue: 4,pp 733737,January 2012

[11] Zia Ul Mahmood et al," Implementation of Spacetime Adaptive Processing (STAP) for Target Detection in Passive Bi-static Radar", PIERS Proceedings, Kuala Lumpur, MALAYSIA, March 27-30, 2012

[12] Jin Yonggao et al, "HF radar sea clutter rejection by nonlinear projections", IEEE Journal of Systems Engineering and Electronics, Volume: 16, Issue: 4,pp. $733-737$.

[13] Kandar et al, "Implementation of MTI based pulse compression Radar system using DSRC communication channel", International Journal of Information Technology and Knowledge Management,Vol.2,pp.379-382,July-December 2010 .
[14] R. Vicen-Bueno et al, "Artificial Neural NetworkBased Clutter Reduction Systems for Ship Size Estimation in Maritime Radars", EURASIP Journal on Advances in Signal Processing, Vol.2010, doi:10.1155/2010/380473.

[15] P.Vouras," Detection of Targets in Band limited and Spatially Correlated Clutter", 2006 IEEE Conference on Radar,pp.8,doi:10.1109/RADAR.2006.1631884,24 -27April,2006.

Priyabrata Karmakar born in Katihar in the state of Bihar,India, He received his B.Tech and M.Tech in Electronics \& Communication Engineering from West Bengal University of Technology and Sikkim Manipal University respectively. He is Assistant Professor at the department of Electronics \& Communication Engineering, Surendra Institute of Engineering \& Management, Siliguri, India.

Sourav Dhar born at Raiganj, West Bengal, INDIA. $\mathrm{He}$ received B.E from Bangalore institute of Technology and M.Tech from Sikkim Manipal Institute of Technology. Currently working as Associate Professor, Department of E\&C Engineering, Sikkim Manipal University, Sikkim, India.

Mithun Chakaraborty born in Jalpaiguri the state of West Bengal, India.He received his B.Tech and M.Tech in Electronics \& Communication Engineering from North Bengal University and Sikkim Manipal University respectively. He is Assistant Professor (Sr.) at the department of Electronics \& Communication Engineering, Surendra Institute of Engineering \& Management, Siliguri, India.

Tirthankar Paul born in Jalapaiguri in the state of West Bengal, India. He received his B.Tech and M.Tech in Electronics \& Communication Engineering from West Bengal University of Technology and Sikkim Manipal University respectively. $\mathrm{He}$ is Assistant Professor at the department of Electronics \& Communication Engineering, Swami Vivekanada Institute of Science \& Technology,Kolkata,India. 